\title{
On the Boltzmann Equation in Unbounded Space far from Equilibrium, and the Limit of Zero Mean Free Path
}

\author{
Leif Arkeryd \\ Department of Mathematics, Chalmers University of Technology and University of Göteborg, S-412 96 \\ Göteborg, Sweden
}

\begin{abstract}
This paper studies Loeb solutions of the Boltzmann equation in unbounded space under natural initial conditions of finite mass, energy, and entropy. An existence theory for large initial data is presented. Maxwellian behaviour is obtained in the limits of zero mean free path and of infinite time. In the standard, space-homogeneous, hard potential case, the infinite time limit is of strong $L^{1}$ type.
\end{abstract}

\section{Preliminaries}

This paper considers Boltzmann's equation, a prototype model of rarefied neutral gases driven by binary collisions. For gases in full space or bounded containers, various contraction mapping estimates can be used to prove the existence of unique, smooth solutions converging to an equilibrium with time, if the initial values are close enough to this equilibrium. Such methods break down, when the initial values are further away from equilibrium, and so do natural compactness arguments, at least in the space-dependent case. For another approach, recall that what happens at distances or within volumes below, say, the scale of elementary particle phenomena, is an artefact of the model with little direct experimental relevance. In this perspective, the question whether the model starts from an underlying set of rationally, really, or infinitesimally spaced points, should be decided purely on mathematical grounds.

In this paper we study the Boltzmann gases on a three-dimensional continuum filled with a denser set of points than the usual triples of reals, in that a non-standard extension of the reals is used instead. In such a setting an integrated form of the actual Boltzmann equation can be solved for arbitrary initial mass distributions with finite initial entropy and second moments. Just like all classical computational models, whatever interesting physical quantities there are, such as moments, they correspond also in the present context to real-valued integrals of the solutions multiplied by test functions. Our solutions display a weak form of Maxwellian behaviour in the small mean free path limit. Globally bounded moments of some 
order $s>2$ imply stronger limiting behaviour. In particular standard spaceindependent solutions under intermolecular forces of hard type, converge strongly in $L^{1}$ to the relevant Maxwellian, when $t \rightarrow \infty$.

The rest of the present section is devoted to a short presentation of the Boltzmann equation and a truncated solution. In Sect. 2 integrated Loeb solutions are proved to exist in the unbounded space case, and their large-time behaviour is analysed. Section 3 demonstrates a limiting Maxwellian behaviour, when the mean free path tends to zero. The final section is devoted to standard solutions in the space-homogeneous case, and proves strong $L^{1}$-convergence to the relevant Maxwellian, if initially there exist entropy and moments of some order $s>2$.

An introduction to non-standard analysis, stressing applications in mathematical physics can be found in e.g. [AFHL]. For a very brief introduction aiming at a gaskinetic context, see [A3]. Readers not familiar with non-standard analysis may benefit from the following informal description of some of the non-standard concepts used in the present paper. The hyperreals ${ }^{*} R$ is a proper, ordered field extension of the reals $R$. They can be imagined as the reals with a monad of infinitesimals added around each real, and with the inverses of the infinitesimals adjoined at $\pm \infty$. The standard part of $b \epsilon^{*} R$ is

$$
\text { st } b={ }^{o} b=\left\{\begin{array}{l}
c \text { if } b \in *^{*} R, c \in R, b-c \text { infinitesimal, } \\
+\infty \text { if } b>n \text { for every } n \in N \\
-\infty \text { if } b<-n \text { for every } n \in N
\end{array}\right.
$$

The number $b$ is said to be finite or near-standard (ns), if $s t b$ is real.

We can think of classical analysis as the study of $V(R)$, where

$$
V_{1}(R)=R, \quad V_{n+1}(R)=V_{n}(R) U\left\{X ; X \subseteq V_{n}(R)\right\}, \quad V(R)=\bigcup_{n<\infty} V_{n}(R) .
$$

Non-standard analysis is the study of the corresponding $V\left({ }^{*} R\right)$, together with a mapping $*: V(R) \rightarrow V\left({ }^{*} R\right)$, such that i)* $r=r$ for $r \in R$; ii) an elementary statement is true of $S_{1}, \ldots, S_{n}$ in $V(R)$, if and only if it is true of ${ }^{*} S_{1}, \ldots,{ }^{*} S_{n}$ in $V\left({ }^{*} R\right)$. From the transfer principle ii), we can see that non-standard analysis is naturally concerned about *images of sets in $V(R)$, so-called standard sets, and set-elements of such sets, so-called internal sets. Non-standard analysis as such has nothing to say about other subsets of $V\left({ }^{*} R\right)$, the so-called external sets. In particular, $N \subseteq{ }^{*} N$ is external, and with it countable unions of internal sets. It follows that the finitely additive * Lebesgue measure is not countably additive, only *countably so. This necessitates some "extension of the *Lebesgue measure" to get a classical Lebesgue measure based on the new denser set ${ }^{*} R^{n}$, the so-called Loeb measure. The basic construction in the present paper uses * Lebesgue solutions of truncated equations to obtain Loeb solutions of the actual Boltzmann equation.

The Boltzmann equation describes the evolution of densities $F$ in phase space, which in this paper is taken essentially as $R^{3} \times R^{3}$. The amount of matter in a region $Y$, is then given by $\int_{Y} F(x, v, t) d x d v$. As is well known, Boltzmann expressed the material derivative $D_{t} F$ through a balance between the density of molecules entering a region of collision and of those leaving it, in the absence of exterior forces as

$$
\partial_{t} F\left(x, v_{1}, t\right)+v_{1} \cdot \nabla_{x} F\left(x, v_{1}, t\right)=Q F\left(x, v_{1}, t\right) / \varepsilon \quad(t>0) .
$$


Here $\nabla_{x}$ is the gradient with respect to the position $x \in R^{3}, \varepsilon$ is the mean free path, and $Q$ is the collision operator,

$$
Q F\left(x, v_{1}\right)=\int_{R^{3} \times B}\left(F\left(x, v_{1}^{\prime}\right) F\left(x, v_{2}^{\prime}\right)-F\left(x, v_{1}\right) F\left(x, v_{2}\right)\right) k\left(v_{1}, v_{2}, u\right) d v_{2} d u
$$

here sometimes abbreviated to $Q F\left(x, v_{1}\right)=\int\left(F \otimes F^{\prime}-F \otimes F\right) k d v_{2} d u$. Given two molecules of initial velocities $v_{1}, v_{2}$, and initially separated in space, $v_{1}^{\prime}, v_{2}^{\prime}$ are the velocities of the molecules after collision. The parameter set $B=\{u=(\theta, \phi)$; $0 \leqq \theta \leqq \pi / 2,0 \leqq \phi \leqq 2 \pi\}$ describes the details of the collision process. A general discussion of $Q$ and $B$ can be found in e.g. [C, TM].

For inverse $j^{t h}$ power intermolecular forces, $j>1, k=\left|v_{2}-v_{1}\right|^{(j-5) /(j-1)} \beta(\theta)$, where $\beta(\theta) \sim|\pi / 2-\theta|^{-(j+1) /(j-1)}$ as $\theta \uparrow \pi / 2$. For the convergence of $\int_{B} k\left(v_{1}, v_{2}, u\right) d u$, we here assume that $k\left(v_{1}, v_{2}, u\right)=k_{1}\left(\left|v_{2}-v_{1}\right|\right) \beta(\theta)$, where $\beta$ is measurable and bounded, and (for Sect. 3 ) locally bounded from below by positive constants on the interior of $(0, \pi / 2)$. We also take $0<k_{1}(w) \leqq C \cdot\left(w^{-\gamma}+1+w^{\lambda}\right)$ for $w \neq 0$, with $0 \leqq \lambda<2,0 \leqq \gamma<3$, where the condition $\gamma<3$ is used to make $k\left(v_{1}, v_{2}, u\right)$ locally integrable, and $\lambda<2$ to control $F\left(x, v_{1}\right) k\left(v_{1}, v_{2}, u\right)$ for large $v_{1}$.

In the discussion below we also use a truncated version $k^{n}$ of $k$. With $a \wedge b=$ $\min (a, b)$, set

$$
\begin{aligned}
& k^{n}\left(v_{1}, v_{2}, u\right)=k\left(v_{1}, v_{2}, u\right) \wedge n \text { for } u \in B \text { and } v_{1}^{2}+v_{2}^{2} \leqq n^{2}, \\
& k^{n}\left(v_{1}, v_{2}, u\right)=0 \text { otherwise, } \\
& \chi(s)=0 \text { for } s \leqq 0, \chi(s)=1 \text { for } s \geqq 1,0 \leqq \chi \leqq 1, \text { and } \chi \in C^{\infty}(R),
\end{aligned}
$$

and define

$$
k^{n}\left(x, v_{1}, v_{2}, u\right)=\chi\left(n^{3}-|x|^{2}\right) \bar{k}^{n}\left(v_{1}, v_{2}, u\right) .
$$

The corresponding integrated Boltzmann equation is

$$
F\left(x+t v_{1}, v_{1}, t\right)=F_{0}\left(x, v_{1}\right)+\int_{0}^{t} Q_{n} F\left(x+s v_{1}, v_{1}, s\right) d s / \varepsilon
$$

with

$$
\begin{aligned}
Q_{n} F\left(x, v_{1}\right)= & \int_{\substack{R^{3} \times B\\
}}\left(\left\langle F\left(x, v_{1}^{\prime}\right) F\left(x, v_{2}^{\prime}\right)\right\rangle-\left\langle F\left(x, v_{1}\right) F\left(x, v_{2}\right)\right\rangle\right) \\
& \cdot k^{n}\left(x, v_{1}, v_{2}, u\right) d v_{2} d u
\end{aligned}
$$

and

$$
\begin{aligned}
& \left\langle F\left(x, v_{1}\right) F\left(x, v_{2}\right)\right\rangle=F\left(x, v_{1}\right) F\left(x, v_{2}\right) \text { if }\left|F\left(x, v_{1}\right) F\left(x, v_{2}\right)\right| \leqq n, \\
& \left\langle F\left(x, v_{1}\right) F\left(x, v_{2}\right)\right\rangle=n \operatorname{sign} F\left(x, v_{1}\right) F\left(x, v_{2}\right) \text { otherwise. }
\end{aligned}
$$

Equation (1.1) is easy to solve, since the truncated collision operator $Q_{n}$ is locally Lipschitz continuous in $L^{\infty}$.

Proposition [A2]. For any initial value $F_{0}$ in the positive cone of $L^{\infty}\left(R^{6}\right)$, there exists a unique, non-negative solution $F$ of (1.1). If

$$
F_{0}, x^{2} F_{0}, v^{2} F_{0}, F_{0} \ln F_{0} \in L^{1}\left(R^{6}\right)
$$


then the integrals

$$
\int F(x, v, t) d x d v, \int F(x, v, t) v^{2} d x d v, \int F(x+v t, v, t) x^{2} d x d v
$$

are conserved, and the integral $\int F(x, v, t) \ln F(x, v, t) d x d v$ is non-increasing.

\section{Loeb Solutions}

By transfer the truncated existence result of the previous section also holds in the non-standard context for $n \epsilon^{*} N-N$. In the case of bounded entropy and bounded second moments, the corresponding solution $f$ is $S$-integrable, thus ${ }^{0} f$ is Loeb integrable. A closer analysis leads to the result that in (1.1) $\langle f \otimes f\rangle$ can be substituted by ${ }^{0} f \otimes{ }^{0} f, k^{n}$ by ${ }^{0} k$, and ${ }^{*} R^{3}$ by $n s^{*} R^{3}$, if we use Loeb integration.

Theorem 2.1. Let $f$ be the solution of the non-standard truncated Boltzmann equation (1.1) with $n \in{ }^{*} N-N,{ }^{o} \varepsilon>0$, and initial condition

$$
f(x, v, 0)={ }^{*} F_{o}(x, v) \wedge n+n^{-1} \exp \left(-v^{2}-x^{2}\right)=f_{o}(x, v),
$$

where $F_{o}$ satisfies (1.2). Then ${ }^{o}\left(1+v^{2}+x^{2}\right) f(x, v, t) \in \operatorname{Loeb} L^{1}\left(n s^{*} R^{6}\right)$ for $t \in n s^{*} R_{+} \cdot{ }^{o} f$ is a Loeb solution of the integrated Boltzmann equation; for Loeb a.e. $\left(x, v_{1}\right) \in n s^{*} R^{6}$,

$$
\begin{aligned}
& { }^{o} f\left(x+t v_{1}, v_{1}, t\right)=F_{o} \circ s t\left(x, v_{1}\right) \\
& \quad+\int_{0_{n s^{*} R^{3} \times B}^{t}} \int_{0^{3}}{ }^{o} f\left(x+s v_{1}, v_{1}^{\prime}, s\right)^{o} f\left(x+s v_{1}, v_{2}^{\prime}, s\right) k^{\circ} s t\left(v_{1}, v_{2}, u\right) L d v_{2} d u d s / \varepsilon \\
& \quad-\int_{0^{*} R^{3} \times B} \int^{o} f\left(x+s v_{1}, v_{1}, s\right)^{o} f\left(x+s v_{1}, v_{2}, s\right) k^{\circ} s t\left(v_{1}, v_{2}, u\right) L d v_{2} d u d s / \varepsilon,
\end{aligned}
$$

when $t \in n s^{*} R_{+}$. The solution conserves mass and first moments in $v$ and $(x-v t)$, has globally bounded $H$-function, and satisfies

$$
\begin{aligned}
\int_{n s^{*} R^{6}}{ }^{o} v^{2 o} f(x, v, t) L d x d v & \leqq \int_{R^{6}} v^{2} F_{o}(x, v) d x d v, \\
\int_{n s^{*} R^{6}}{ }^{o} x^{2 o} f(x+t v, v, t) L d x d v & \leqq \int_{R^{6}} x^{2} F_{o}(x, v) d x d v .
\end{aligned}
$$

Remark. Corresponding results have been obtained in the space-periodic case [A3], and for bounded $C^{1}$-regions with reflection type boundary conditions and exterior forces [E]. A wider class of non-negative, * Lebesgue-measurable initial data can also be handled with the same methods. The theorem also holds for radial cut-off in $k$.

Proof. For $t \geqq 0, f$ is $S$-integrable as a function of $x$ and $v$. This is a direct consequence of "infinitesimal mass from infinite function values,"

$$
\int_{R^{6}}(f(t)-f(t) \wedge \omega)^{*} d x d v \leqq(\log \omega)^{-1} \int_{R^{6}} f(t) \log ^{+} f(t)^{*} d x d v \approx 0\left(\omega \in^{*} N-N\right),
$$

and of "infinitesimal mass from infinitesimal function values,"

$$
\begin{gathered}
\int_{R^{6}} f(t) \wedge \omega^{-1 * d x d v \leqq} \int_{x^{2}+v^{2} \leqq \omega^{1 / 4}} \omega^{-1} * d x d v+\left(1+\omega^{1 / 4}\right)^{-1} \\
\int_{R^{6}}\left(1+x^{2}+v^{2}\right) f(t)^{*} d x d v \approx 0 \quad\left(\omega \in^{*} N-N\right) .
\end{gathered}
$$


Moreover, for Loeb a.e. $\left(x, v_{1}\right) \in n s^{*} R^{6}$,

$$
\begin{aligned}
& \int_{0}^{o t} \int_{R_{R^{3} \times B}}\langle f \otimes f\rangle\left(x+s v_{1}, v_{1}, v_{2}, s\right) k^{n}\left(x, v_{1}, v_{2}, u\right)^{*} d v_{2} d u d s \\
& \quad=\int_{0_{n s^{*} R^{3} \times B}}^{t} \int_{0} f\left(x+s v_{1}, v_{1}, s\right)^{o} f\left(x+s v_{1}, v_{2}, s\right) k^{\circ} s t\left(v_{1}, v_{2}, u\right) L d v_{2} d u d s
\end{aligned}
$$

and

$$
\begin{aligned}
& \int_{0}^{o t} \int_{R^{3} \times B}\langle f \otimes f\rangle\left(x+s v_{1}, v_{1}^{\prime}, v_{2}^{\prime}, s\right) k^{n}\left(x, v_{1}, v_{2}, u\right)^{*} d v_{2} d u d s \\
& \quad=\int_{0_{n s^{*} R^{3} \times B}}^{t} \int^{o} f\left(x+s v_{1}, v_{1}^{\prime}, s\right)^{o} f\left(x+s v_{1}, v_{2}^{\prime}, s\right) k^{\circ} s t\left(v_{1}, v_{2}, u\right) L d v_{2} d u d s .
\end{aligned}
$$

Equations (2.2) and (2.3) follow from the corresponding proofs in [A3, E], adapted to the present situation of unbounded $x$-variable. That ${ }^{o} f$ is a solution of $(2.1)$ is an immediate consequence of (2.2) and (2.3). Since $f(t)$ is $S$-integrable for $t>0$ with bounded second moments, it follows that ${ }^{\circ} f(t)\left(1+x^{2}+v^{2}\right) \in \operatorname{Loeb} L^{1}\left(n s^{*} R^{6}\right)$. The conservation of mass and first moments, as well as the bounds on $x^{2}$ - and $v^{2}$ moments, are immediate consequences of the truncated results of the previous section. It remains to prove that the $H$-function is globally bounded;

$$
\begin{aligned}
& \int_{n s^{*} R^{6}} f(t) \ln o f(t) L d x d v \leqq \int_{n s^{*} R^{6}} f^{o} f(t) \ln ^{+o} f(t) L d x d v \\
& \leqq \\
& \int_{* R^{6}}^{o} f(t) \ln ^{+} f(t)^{*} d x d v \leqq \int_{R^{6}}^{o} f(t) \ln f(t)^{*} d x d v \\
& \quad+\int_{R^{6}}^{o} f(t) \ln ^{-} f(t)^{*} d x d v \leqq \int_{R^{6}}^{o} f_{o} \ln f_{o}^{*} d x d v \\
& \quad+\int_{R^{6}}^{o} \exp \left(-x^{2}-v^{2}\right)^{*} d x d v+\int_{R^{6}}^{o}\left(x^{2}+v^{2}\right) f(x+v t, v, t)^{*} d x d v \\
& =\int_{R^{6}} F_{o} \ln F_{o} d x d v+\int_{R^{6}} \exp \left(-x^{2}-v^{2}\right) d x d v+\int_{R^{6}}\left(x^{2}+v^{2}\right) F_{o} d x d v .
\end{aligned}
$$

Thus the $H$-function is globally bounded.

In the full-space case of Theorem 2.1 the mass spreads out over $n s^{*} R^{3}$, as $t \rightarrow \infty$. We have

Theorem 2.2. Suppose $n \in N$, and define

$$
A\left(x_{o}\right)=\left\{(x, v) \in^{*} R^{3} \times R^{3} ;\left|x-x_{o}\right| \leqq n, v \in n s^{*} R^{3}\right\} .
$$

Then under the conditions of Theorem 2.1,

$$
\lim _{o_{t} \rightarrow \infty} \sup _{x_{o} \in n s^{*} R^{3}} \int_{A\left(x_{o}\right)}{ }^{o} f(x, v, t) L d x d v=0 .
$$

Proof. The boundedness of energy implies

$$
\int_{|v|>j}{ }^{o} f(x, v, t) L d x d v \leqq\left(1+j^{2}\right)^{-1} \int_{R^{6}}\left(1+v^{2}\right) F_{o}(x, v) d x d v \quad(t>0) .
$$


This tends to zero when $j \rightarrow \infty$, so it is enough to prove

where

$$
\lim _{o_{t \rightarrow \infty}} \sup _{x_{o} \in n^{*} R^{3}} \int_{A_{1}\left(x_{o}, j\right)}{ }^{o} f(x, v, t) L d x d v=0 \quad(j \in N)
$$

But

$$
A_{1}\left(x_{o}, j\right)=\left\{(x, v) \varepsilon^{*} R^{3} \times R^{3} ;\left|x-x_{0}\right| \leqq n, v \varepsilon n s^{*} R^{3},|v| \leqq j\right\}
$$

$$
\int_{A_{2}\left(x_{o}, \delta\right)}{ }^{o} f(x, v, t) L d x d v \leqq \delta^{2} \pi^{2} n^{3} 16 / 9-\int_{n s^{*} R^{6}}{ }^{o} f(x, v, t) \ln ^{+} f(x, v, t) L d x d v / \ln \delta,
$$

where

$$
A_{2}\left(x_{o}, \delta\right)=\left\{(x, v) \varepsilon^{*} R^{3} \times R^{3} ;\left|x-x_{o}\right| \leqq n,\left|v-x_{o} / t\right| \leqq \delta\right\} .
$$

This tends to zero (uniformly in $t$ ), when $\delta \rightarrow 0$.

Finally, set

$$
A_{3}\left(x_{o}, \delta\right)=\left\{(x, v) \varepsilon^{*} R^{3} \times R^{3} ;\left|x-x_{o}\right| \leqq n, \delta \leqq\left|v-x_{o} / t\right|,|v| \leqq j\right\} .
$$

Then for $t \delta>n / \delta+n$,

$$
\begin{aligned}
\int_{A_{3}\left(x_{o}, \delta\right)} o f(x, v, t) L d x d v & \leqq(\delta / n)^{2} \int_{A_{3}\left(x_{o}, \delta\right)} o^{o}|x-t v|^{2} f(x, v, t) L d x d v \\
& \leqq(\delta / n)^{2} \int_{R^{3}} x^{2} F_{o}(x, v) d x d v
\end{aligned}
$$

which tends to zero, when $\delta \rightarrow 0$. This completes the proof of the theorem.

\section{The Limit of Vanishing Mean Free Path}

Theorem 2.1 was proved for non-infinitesimal mean free paths, whereas for the nonstandard function $f$ defined in that theorem, its standard part ${ }^{\circ} f$ is Loeb integrable, also when $\varepsilon>0$ is infinitesimal, $\varepsilon \approx 0$. We write $f=f_{\varepsilon}$ to stress the dependence of the solution on the mean free path in this section.

The case of infinitesimal $\varepsilon$ contains the behaviour of classical weak $L^{1}$ cut-off limits in the following sense. The Loeb solution ${ }^{o} f_{\varepsilon}(t)$ defines an $L^{1}$-density $T f_{\varepsilon}(t)$ through

$$
\int_{n s^{*} R^{6}}{ }^{o} f_{\varepsilon}(x, v, t) \phi \circ s t(x, v) L d x d v=\int_{R^{6}} T f_{\varepsilon}(x, v, t) \phi(x, v) d x d v \quad\left(\phi \in C_{0}\left(R^{6}\right)\right) .
$$

Suppose $\left(f_{\varepsilon_{j}}^{n_{j}}\right)_{j \in N}$ is a weakly convergent sequence of $L^{1}$-solutions of $(1.1)$ with $\left(n_{j}\right)_{j \in N}$ a sequence of standard reals increasing to $\infty$, with $\left(\varepsilon_{j}\right)_{j \in N}$ a sequence of standard reals decreasing to 0 , and with initial values

$$
F_{o} \wedge n_{j}+\left(n_{j}\right)^{-1} \exp \left(-v^{2}-x^{2}\right) .
$$

By transfer there is $\varepsilon \approx 0, n \in^{*} N-N$, such that the $f_{\varepsilon}$ of Theorem 2.1 corresponding to a truncation at $n$, generates the limit density $T f_{\varepsilon}$,

$$
\int_{R^{6}} T f_{\varepsilon}(x, v, t) \phi(x, v) d x d v=\lim _{j \rightarrow \infty} \int_{R^{6}} f_{\varepsilon_{j}}(x, v, t) \phi(x, v) d x d v \quad\left(\phi \in C_{o}\left(R^{6}\right)\right) .
$$


Moreover, any $f_{\varepsilon}$ with $\varepsilon \approx 0$, is infinitesimally close to a local Maxwellian, as we shall now prove.

Lemma 3.1. When $\varepsilon \approx 0$, it holds for Loeb a.e. $(x, t) \in\left(n s^{*} R^{3}\right) x^{*} R_{+}$, that

$$
\begin{aligned}
& f_{\varepsilon}\left(x, v_{1}, t\right) f_{\varepsilon}\left(x, v_{2}, t\right) \approx f_{\varepsilon}\left(x, v_{1}^{\prime}, t\right) f_{\varepsilon}\left(x, v_{2}^{\prime}, t\right) \\
& \text { for Loeb a.e. }\left(v_{1}, v_{2}, u\right) \in n s^{*} R^{3} \times R^{3} \times B .
\end{aligned}
$$

Proof. By *Gibbs' lemma,

$$
\inf \left\{\int_{R^{6}} f_{\varepsilon}(x, v, t) \ln f_{\varepsilon}(x, v, t)^{*} d x d v ; t \geqq 0\right\}
$$

is finite. It follows that for infinitesimal mean free path, $\varepsilon \approx 0$,

$$
\begin{aligned}
& 0 \leqq \int\left(\left\langle f_{\varepsilon}^{\prime} \otimes f_{\varepsilon}^{\prime}\right\rangle-\left\langle f_{\varepsilon} \otimes f_{\varepsilon}\right\rangle\right) \ln \left(f_{\varepsilon}^{\prime} \otimes f_{\varepsilon}^{\prime} / f_{\varepsilon} \otimes f_{\varepsilon}\right) k^{n}\left(x, v_{1}, v_{2}, u\right)^{*} d x d v_{1} d v_{2} d u d 4^{-1} \\
& \quad=-\varepsilon \int f_{\varepsilon}(x, v, t) \ln f_{\varepsilon}(x, v, t)^{*} d x d v+\varepsilon \int f_{o}(x, v) \ln f_{o}(x, v)^{*} d x d v \approx 0 .
\end{aligned}
$$

So for Loeb a.e. $(x, t) \in\left(n s^{*} R^{3}\right) x^{*} R_{+}$, it holds that

$$
\begin{aligned}
& 0 \leqq\left(\left\langle f_{\varepsilon}^{\prime} \otimes f_{\varepsilon}^{\prime}\right\rangle-\left\langle f_{\varepsilon} \otimes f_{\varepsilon}\right\rangle\right) \ln \left(f_{\varepsilon}^{\prime} \otimes f_{\varepsilon}^{\prime} / f_{\varepsilon} \otimes f_{\varepsilon}\right) \approx 0 \\
& \text { for Loeb a.e. }\left(v_{1}, v_{2}, u\right) \in n s^{*} R^{3} \times R^{3} \times B .
\end{aligned}
$$

And so for these $\left(v_{1}, v_{2}, u\right)$,

$$
f_{\varepsilon}^{\prime} \otimes f_{\varepsilon}^{\prime} / f_{\varepsilon} \otimes f_{\varepsilon} \approx 1 \quad \text { or }\left\langle f_{\varepsilon}^{\prime} \otimes f_{\varepsilon}^{\prime}\right\rangle \approx\left\langle f_{\varepsilon} \otimes f_{\varepsilon}\right\rangle .
$$

It follows from the conservation of the finite mass, that for all $t$

$$
\int f_{\varepsilon}(x, v, t)^{*} d v \text { is finite for Loeb a.e. } x \in{ }^{*} R^{3} \text {. }
$$

Hence for Loeb a.e. $x \in R^{*}$,

$$
f_{\varepsilon}\left(x, v_{1}, t\right) f_{\varepsilon}\left(x, v_{2}, t\right), f_{\varepsilon}\left(x, v_{1}^{\prime}, t\right) f_{\varepsilon}\left(x, v_{2}^{\prime}, t\right)
$$

are finite for Loeb a.e. $\left(v_{1}, v_{2}, u\right) \in n s^{*} R^{3} \times R^{3} \times B$. For these $\left(v_{1}, v_{2}, u\right)$ the bracket is redundant in (3.2), and so the lemma follows.

A function which satisfies (3.1) is either Loeb a.e. infinitesimal or Loeb a.e. noninfinitesimal on $n s^{*} R^{3}$. We have

Lemma 3.2. Let $g \in^{*} L_{+}^{1}\left(R^{3}\right)$ be given with

and with

$$
\int g(v)\left(1+v^{2}\right)^{*} d v, \quad \int g(v) \ln g(v)^{*} d v \text { finite, }
$$

$$
g\left(v_{1}\right) g\left(v_{2}\right) \approx g\left(v_{1}^{\prime}\right) g\left(v_{2}^{\prime}\right) \text { for Loeb a.e. }\left(v_{1}, v_{2}, u\right) \in n s^{*} R^{3} \times R^{3} \times B .
$$

Then either

$$
g(v) \approx 0 \text { for Loeb a.e. } v \in n s^{*} R^{3},
$$

or

$$
{ }^{\circ} g(v)>0 \text { for Loeb a.e. } v \in n s^{*} R^{3} \text {. }
$$

Proof. Consider for simplicity a representative from the equivalence class $g$, still (somewhat incorrectly) denoted $g$. The conditions of the lemma imply that $g$ is $S$ integrable. Either $g \approx 0$ for Loeb a.e. $v \in n s^{*} R^{3}$, or there is a *measurable set $A$ of 
finite diameter and non-infinitesimal measure in $n s^{*} R^{3}$, where $g$ is noninfinitesimal. We can choose this set $A$ so that, moreover, $g$ is finite on $A$ with $C<g<1 / C$ for some $C \in R_{+}$.

If ${ }^{o} g>0$ Loeb a.e. on a sphere of non-infinitesimal measure, then the corresponding construction in [A1] shows that ${ }^{\circ} g>0$ Loeb a.e. on $n s^{*} R^{3}$. In any case there is a *finite sequence of disjoint balls $B_{j}, j=1, \ldots, j_{o}$, such that (with $\mu$ the *Lebesgue measure)

$$
\mu\left(A \cap\left(\bigcup_{1}^{j_{o}} B_{j}\right)\right) \approx \mu(A) \approx \mu\left(\bigcup_{1}^{j_{o}} B_{j}\right) .
$$

So if one of the $B_{j}$ 's has non-infinitesimal measure, then the lemma holds. Otherwise $j_{o} \varepsilon * N-N$, and each $B_{j}$ has infinitesimal measure. In this case the lemma follows, if we can construct a ball of non-infinitesimal measure, where ${ }^{\circ} g>0$ Loeb a.e.. The rest of the proof is devoted to that construction.

We first notice, that for some $\eta \approx 0$,

$$
\left|g \otimes g-g^{\prime} \otimes g^{\prime}\right| \leqq \eta \text { in }\left(\bigcup_{1}^{j_{o}} B_{j}\right) \times\left(\bigcup_{1}^{j_{o}} B_{j}\right) x^{*} B,
$$

except on an internal set of *Lebesgue measure less than $\eta$, and

$$
C<g<1 / C \text { in } \bigcup_{1}^{j_{o}} B_{j}
$$

except on an internal set of *Lebesgue measure less than $\eta$. It follows that there are balls $B_{j_{1}}$ and $B_{j_{2}}$, such that for some $v \in * N-N$, the subset of $B_{j_{1}} \times B_{j_{2}} \times{ }^{*} B$, where

$$
\left|g \otimes g-g^{\prime} \otimes g^{\prime}\right|>\eta,
$$

is contained in a ${ }^{*}$ measurable set of measure less than

$$
2^{-v} \mu\left(B_{j_{1}} \times B_{j_{2}} \times{ }^{*} B\right),
$$

and such that the *measurable subsets of $B_{j_{1}}\left(B_{j_{2}}\right)$, where $C<g<1 / C$ does not hold, has measure less than

$$
2^{-v} \mu\left(B_{j_{1}}\right)\left(2^{-v} \mu\left(B_{j_{2}}\right)\right) .
$$

(Below such statements are abbreviated as "outside of a $2^{-v}$-fraction of...." The above holds for one and the same pair $\eta, v$, and a *finite sequence of pairs of balls in $\left(\bigcup_{1}^{j_{o}} B_{j}\right) \times\left(\bigcup_{1}^{j_{o}} B_{j}\right)$. With $I$ its index set, the sequence can be so chosen that

$$
\mu\left(\bigcup_{\left(j_{1}, j_{2}\right) \in I}\left(B_{j_{1}} \times B_{j_{2}} \times{ }^{*} B\right)\right) \approx \mu\left(\left(\bigcup_{1}^{j_{o}} B_{j}\right) \times\left(\bigcup_{1}^{j_{o}} B_{j}\right) \times{ }^{*} B\right) .
$$

For each $\left(j_{1}, j_{2}\right) \in I$

$$
-\eta+C^{2}<g^{\prime} \otimes g^{\prime}<1 / C^{2}+\eta
$$

for all $\left(v_{1}, v_{2}, u\right)$ outside of a $3.2^{-v}$-fraction of $B_{j_{1}} \times B_{j_{2}} \times{ }^{*} B$.

Since ${ }^{o} \mu(A)>0$, it is possible to find $\bar{v}_{1}$, and at a finite, non-infinitesimal distance 
from $\bar{v}_{1}$ a line-segment $I_{1}$, containing a set of points $I_{2}\left(\subseteq I_{1}\right)$ with non-infinitesimal *Lebesgue measure, such that

$$
-\eta+C^{2}<g\left(v_{1}^{\prime}\right) g\left(v_{2}^{\prime}\right)<1 / C^{2}+\eta .
$$

Here $v_{1}^{\prime}, v_{2}^{\prime}$ are given by

$$
\left(v_{1}^{\prime}, v_{2}^{\prime}\right)=\left(v_{1}^{\prime}\left(v_{1}, v_{2}, u\right), v_{2}^{\prime}\left(v_{1}, v_{2}, u\right)\right)
$$

where $v_{1}=\bar{v}_{1}, v_{2} \in I_{2}$, and for each $v_{2}, u$ belongs to a subset $B_{v_{2}} \subseteq{ }^{*} B$ of full Loeb measure. There is a subset $I_{3} \subseteq I_{2}$ of non-infinitesimal ${ }^{*}$ Lebesgue-measure, such that $g\left(v_{2}^{\prime}\right)$ is finite for

$$
v_{2}^{\prime}=v_{2}^{\prime}\left(\bar{v}_{1}, v_{2}, u\right), v_{2} \in I_{3}, u \in \bar{B}_{v_{2}},
$$

where for each $v_{2} \in I_{3}, \bar{B}_{v_{2}}$ has full Loeb measure in ${ }^{*} B$. Evidently $g\left(v_{1}^{\prime}\right)$ is noninfinitesimal for the same points $\left(\bar{v}_{1}, v_{2}, u\right)$. The set

$$
\bigcup_{v_{2} \in I_{3}}\left\{v_{1}^{\prime}\left(\bar{v}_{1}, v_{2}, u\right) ; u \in \bar{B}_{v_{2}}\right\}
$$

has non-infinitesimal *Lebesgue measure. Each subset

$$
T_{v_{2}}=\left\{v_{1}^{\prime}\left(\bar{v}_{1}, v_{2}, u\right) ; u \in \bar{B}_{v_{2}}\right\}, v_{2} \in I_{3},
$$

has non-infinitesimal diameter and full Loeb measure in the sphere

$$
\left\{v_{1}^{\prime}\left(\bar{v}_{1}, v_{2}, u\right) ; u \in * B\right\} \text {. }
$$

We now use the previous line of argument once more. There is a ball $B_{j}$, and a set $T_{\bar{v}_{2}}$ as above, at non-infinitesimal distance from each other, such that $\left|g^{\prime} \otimes g^{\prime}-g \otimes g\right|<\eta$, except for $\left(v_{1}, v_{2}, u\right)$ in an infinitesimal fraction of $B_{j} \times$ $T_{\bar{v}_{2}} \times{ }^{*} B$, and such that $g$ is non-infinitesimal on $B_{j}$ and $T_{\bar{v}_{2}}$ except for an infinitesimal fraction of these sets. It follows that for some $\bar{v}_{j} \in B_{j}, g\left(v_{1}^{\prime}\right) g\left(v_{2}^{\prime}\right)$ is noninfinitesimal, except when $v_{1}^{\prime}, v_{2}^{\prime}$ are given by $\left(v_{1}, v_{2}, u\right) \in\left\{\bar{v}_{j}\right\} \times T_{\bar{v}_{2}} \times{ }^{*} B$, and $\left(v_{2}, u\right)$ belongs to an infinitesimal fraction of the set $T_{\bar{v}_{2}} \times{ }^{*} B$.

Since $g\left(v_{2}^{\prime}\right)$ is finite for Loeb a.e. $v_{2}^{\prime} \in^{*} R^{3}$, and $T_{\bar{v}_{2}}$ has full Loeb measure on a sphere of non-infinitesimal diameter, from here we conclude that $g\left(v_{1}^{\prime}\right)$ is noninfinitesimal Loeb a.e. on a set containing a ball of non-infinitesimal volume. This completes the proof of the lemma.

It is an easy consequence of (3.1) and Lemma 3.2 that $f_{\varepsilon}$ is almost a local Maxwellian.

Theorem 3.3. For $\varepsilon \approx 0$ and Loeb a.e. $(x, t) \in n s\left({ }^{*} R^{3} \times R_{+}\right)$, there are

$$
a(x, t), b(x, t) \in R_{+} \text {, and } c(x, t) \in R^{3},
$$

such that

$$
f_{\varepsilon}(x, v, t) \approx a(x, t) e^{-b(x, t) v^{2}+c(x, t) \cdot v} \text { for Loeb a.e. } v \in n s^{*} R^{3} .
$$

Proof. It is possible to write

$$
v_{1}^{\prime}=v_{1}+q_{1}, v_{2}^{\prime}=v_{1}+q_{2}, v_{2}=v_{1}+q_{1}+q_{2} \text { with } q_{1} \cdot q_{2}=0
$$


(see e.g. [A1] p. 22). Set

$$
M=\left\{\left(q_{1}, q_{2}\right) \in R^{3} \times R^{3} ; q_{1} \cdot q_{2}=0\right\} .
$$

Then the mapping

$$
\left(v_{2}, u\right) \rightarrow\left(q_{1}, q_{2}\right)=\left(v_{1}^{\prime}\left(v_{1}, v_{2}, u\right)-v_{1}, v_{2}^{\prime}\left(v_{1}, v_{2}, u\right)-v_{1}\right)
$$

is bijective from $R^{3} \times B$ onto $M$ with smooth non-zero jacobian determinant, after deleting a set of measure zero from each space. Consider a representative from the equivalence class $f_{\varepsilon}$, still denoted $f_{\varepsilon}$, and set

$$
\bar{\psi}(q)=\ln \left(f_{\varepsilon}\left(x, v_{1}+q, t\right) / f_{\varepsilon}\left(x, v_{1}, t\right)\right) .
$$

It follows from the previous two lemmas, that for Loeb a.e. $(x, t) \in n s^{*} R^{3} \times R_{+}$, either

$$
f_{\varepsilon}(x, v, t) \approx 0 \text { for Loeb a.e. } v \in n s^{*} R^{3},
$$

or for Loeb a.e. $v_{1} \in n s^{*} R^{3}$

$$
0<{ }^{o} f_{\varepsilon}\left(x, v_{1}, t\right)<\infty,{ }^{o} f_{\varepsilon}\left(x, v_{1}, t\right)^{2}\left(f_{\varepsilon}\left(x, v_{1}^{\prime}, t\right) f_{\varepsilon}\left(x, v_{2}^{\prime}, t\right)\right)^{-1}<\infty
$$

for Loeb a.e. $\left(v_{2}, u\right) \in n s^{*} R^{3} \times B$, together with

$$
\bar{\psi}(q) \text { finite for Loeb a.e. } q \in n s^{*} R^{3}, \bar{\psi}\left(q_{1}+q_{2}\right) \approx \bar{\psi}\left(q_{1}\right)+\bar{\psi}\left(q_{2}\right)
$$

for Loeb a.e. $\left(q_{1}, q_{2}\right) \in n s^{*} M$. So for Loeb a.e. $v_{1} \in n s^{*} R^{3}$,

$$
\bar{\psi}\left(q_{1}+q_{2}\right)=\bar{\psi}\left(q_{1}\right)+\bar{\psi}\left(q_{2}\right)+g\left(q_{1}, q_{2}\right),
$$

where $g \approx 0$ for Loeb a.e. $\left(q_{1}, q_{2}\right) \in n s^{*} M$. It follows that we can choose an orthonormal triple $e_{1}, e_{2}, e_{3}$ in ${ }^{*} R^{3}$, so that

$$
\bar{\psi}\left(y_{1} e_{1}+y_{2} e_{2}+y_{3} e_{3}\right)=\bar{\psi}\left(y_{1} e_{1}\right)+\bar{\psi}\left(y_{2} e_{2}\right)+\bar{\psi}\left(y_{3} e_{3}\right)+\bar{g}\left(y_{1} e_{1}+y_{2} e_{2}+y_{3} e_{3}\right) .
$$

Here

$$
\bar{g}\left(y_{1} e_{1}+y_{2} e_{2}+y_{3} e_{3}\right)=g\left(y_{1} e_{1}, y_{2} e_{2}+y_{3} e_{3}\right)+g\left(y_{2} e_{2}, y_{3} e_{3}\right),
$$

and

$$
\bar{g}\left(y_{1} e_{1}+y_{2} e_{2}+y_{3} e_{3}\right) \approx 0 \text { for Loeb a.e. }\left(y_{1}, y_{2}, y_{3}\right) \in n s^{*} R^{3} \text {. }
$$

With

$$
\psi\left(y_{1} e_{1}+y_{2} e_{2}+y_{3} e_{3}\right)=\bar{\psi}\left(y_{1} e_{1}+y_{2} e_{2}+y_{3} e_{3}\right)-\bar{g}\left(y_{1} e_{1}+y_{2} e_{2}+y_{3} e_{3}\right)+3 \bar{g}(0) / 2,
$$

we have

$$
\psi\left(y_{1} e_{1}+y_{2} e_{2}+y_{3} e_{3}\right)=\psi\left(y_{1} e_{1}\right)+\psi\left(y_{2} e_{2}\right)+\psi\left(y_{3} e_{3}\right)\left(\left(y_{1}, y_{2}, y_{3}\right) \epsilon^{*} R^{3}\right) .
$$

So there are $\bar{b} \in * R$ and $\bar{c} \in * R^{3}$, such that

$$
\psi(v)=\bar{b} v^{2}+\bar{c} \cdot v \text { for a.e. } v \in^{*} R^{3}
$$

(see e.g. [A1] p. 26 and transfer). Here $\bar{b}$ and $\bar{c}$ are finite, since $\bar{\psi}$ is finite and $\bar{g}$ infinitesimal Loeb a.e. on $n s^{*} R^{3}$. So with $b=-{ }^{o} \bar{b}$, and $c={ }^{o} \bar{c}$, we have

$$
e^{\Psi(v)} \approx e^{-b v^{2}+c . v} \text { for Loeb a.e. } v \in n s^{*} R^{3} \text {. }
$$


Hence

$$
f_{\varepsilon}\left(x, v_{1}+v, t\right) \approx f_{\varepsilon}\left(x, v_{1}, t\right) e^{-b v^{2}+c \cdot v} \approx a e^{-b v^{2}+c \cdot v}
$$

for Loeb a.e. $v \in n s^{*} R^{3}$, and with $a={ }^{o} f_{\varepsilon}\left(x, v_{1}, t\right)$. Together with a change of variables, $v \rightarrow v_{1}+v$, this completes the proof of the theorem.

Remark. For $\phi \in{ }^{*} C_{o}^{1}\left(R^{3} \times R_{+}\right)$,

$$
\int_{R^{6}} \int_{0}^{t} Q_{n} f_{\varepsilon}\left(x+s v_{1}, v_{1}, s\right) \phi\left(x+s v_{1}, s\right)^{*} d s^{*} d x d v_{1}=0 .
$$

If the ${ }^{*} C_{0}^{1}$-norm of $\phi$ is finite, then the function $f_{\varepsilon}$ of Theorem 2.1 satisfies (also for infinitesimal $\varepsilon>0$ )

$$
\begin{gathered}
\int_{n s^{*} R^{6}}{ }^{o} f_{\varepsilon}\left(x, v_{1}, t\right)^{o} \phi(x, t) L d x d v_{1}=\int_{n s^{*} R^{6}} F_{0}{ }^{\circ} s t\left(x, v_{1}\right)^{o} \phi(x, 0) L d x d v_{1} \\
+\int_{n s^{*} R^{6} \times[0, t]}{ }^{o} f_{\varepsilon}\left(x, v_{1}, s\right)\left({ }^{o} \partial_{s} \phi(x, s)+{ }^{o} v_{1} \cdot \nabla_{x} \phi(x, s)\right) L d x d v_{1} d s .
\end{gathered}
$$

Inserting (3.3) into (3.4), we obtain the first of the five compressible Euler equations. If $S$-integrability were known for the second and third $v$-moments of $f_{\varepsilon}$, then the other four Euler equations would also follow. In particular this is the case, when the $v^{4}$-moment of $f_{\varepsilon}(t)$ is bounded for $t>0$.

ii) Essentially as a consequence of Theorem 3.3, for $\varepsilon \approx 0$, and Loeb a.e. $(x, t) \in n s^{*} R^{3} \times R_{+}$,

$$
\begin{aligned}
\int_{n s^{*} R^{3}}{ }^{o} f_{\varepsilon}(x, v, t) L d v & =\int_{R^{3}} a(x, t) \exp \left(-b(x, t) v^{2}+c(x, t) \cdot v\right) d v=\int_{R_{R^{3}}}^{o} f_{\varepsilon}(x, v, t)^{*} d v, \\
\int_{n s^{*} R^{3}}{ }^{o} v f(x, v, t) L d v & =\int_{R^{3}} v a(x, t) \exp \left(-b(x, t) v^{2}+c(x, t) \cdot v\right) d v=\int_{*_{R^{3}}}^{o} v f_{\varepsilon}(x, v, t)^{*} d v, \\
\int_{n s^{*} R^{3}}{ }^{o} v^{2} f_{\varepsilon}(x, v, t) L d v & =\int_{R^{3}} v^{2} a(x, t) \exp \left(-b(x, t) v^{2}+c(x, t) \cdot v\right) d v .
\end{aligned}
$$

iii) The result of Theorem 3.3 also holds in the space-periodic case of [A3].

\section{The Space-Homogeneous Standard Boltzmann Equation}

This section discusses the space-homogeneous standard Boltzmann equation under the additional (cf. Sect. 1) restriction on $k_{1}$,

$$
0 \leqq k_{1}(w) \leqq C\left(1+w^{\lambda}\right) \text { with } 0 \leqq \lambda<2 .
$$

This includes inverse $j^{\text {th }}$ power intermolecular forces with angular cut-off and $j>5$. Using the technique of Sect. 3, we shall obtain strong $L^{1}$-convergence to the relevant Maxwellian, when $t \rightarrow \infty$. For large data and $j>5$, previously employed methods only imply weak $L^{1}$-convergence.

We begin by recalling some well-known results about the space-homogeneous, standard Boltzmann equation. The technique of [A1] p. 15 implies the following proposition. 
Proposition 4.1. Assume that

$$
F_{o} \ln F_{o} \in L^{1}\left(R^{3}\right),\left(1+|v|^{s}\right) F_{o} \in L_{+}^{1}\left(R^{3}\right) \text { for some } s>2 .
$$

Then there exists a solution

$$
F: R_{+} \rightarrow L_{+}^{1}\left(R^{3}\right)
$$

of

$$
\partial_{t} F=Q F \quad(t>0) \text { with }\left.F\right|_{t=0}=F_{o}
$$

such that

$$
\int g(v) F(v, t) d v=\int g(v) F_{0}(v) d v \quad(t>0)
$$

for $g=1, v, v^{2}$. Moreover, for any $t_{1}>0$

$$
\int\left(1+|v|^{s}\right) F(v, t) d v \leqq C_{t_{1}} \int\left(1+|v|^{s}\right) F_{o}(v) d v \quad\left(0 \leqq t \leqq t_{1}\right)
$$

with $C_{t_{1}}$ only depending on

$$
\int\left(1+v^{2}\right) F_{o}(v) d v, t_{1}, k, \text { and } s .
$$

The solution is for $t>0$ the weak $L^{1}$-limit of the (unique) solutions of

$$
\partial_{t} F_{v}=Q_{v} F_{v}(t>0) \text { with } F_{v \mid t=0}=F_{o} .
$$

Here the kernel of $Q_{v}$ uses $k_{1} \wedge n_{v}$ instead of $k_{1}$, and $\left(n_{v}\right)_{v \in N}$ is a certain increasing sequence of natural numbers.

The following inequality is due to Elmroth.

Proposition 4.2. [E] For $s>2$, there are $K_{s}>0$, and $C_{s}>0$, such that

$$
\begin{aligned}
& \left|v_{1}^{\prime}\right|^{s}+\left|v_{2}^{\prime}\right|^{s}-\left|v_{1}\right|^{s}-\left|v_{2}\right|^{s} \leqq K_{s}\left(\left|v_{1}\right|^{s-1}\left|v_{2}\right|+\left|v_{1}\right|\left|v_{2}\right|^{s-1}\right) \\
& \cos \theta \sin \theta-C_{s}\left(\left|v_{1}\right|^{s}+\left|v_{2}\right|^{s}\right) \cos ^{2} \theta \sin ^{2} \theta .
\end{aligned}
$$

This estimate can be used to obtain global bounds of higher moments for solutions of the present Boltzmann equation.

Lemma 4.3. The solutions $F_{v}$ of Proposition 4.1 have globally bounded moments of order $s$, and

$$
\sup _{v} \sup _{t>0} \int\left(1+|v|^{s}\right) F_{v}(v, t) d v<\infty \text {. }
$$

Proof. Using Proposition 4.2 we get

$$
\begin{aligned}
& \partial_{t} \int_{R^{3}}|v|^{s} F_{v}(v, t) d v=\frac{1}{2} \int_{R^{6} \times B}\left(\left|v_{1}^{\prime}\right|^{s}+\left|v_{2}^{\prime}\right|^{s}-\left|v_{1}\right|^{s}-\left|v_{2}\right|^{s}\right) F\left(v_{1}, t\right) F\left(v_{2}, t\right) \\
& \cdot k\left(v_{1}, v_{2}, u\right) d v_{1} d v_{2} d u \leqq K_{s} \int_{R^{6} \times B}\left(\left|v_{1}\right|^{s-1}\left|v_{2}\right| \cos \theta \sin \theta F_{v}\left(v_{1}, t\right) F_{v}\left(v_{2}, t\right)\right. \\
& \cdot k\left(v_{1}, v_{2}, u\right) d v_{1} d v_{2} d u-\frac{1}{2} C_{s} \int_{R^{6} \times B}\left(\left|v_{1}\right|^{s}+\left|v_{2}\right|^{s}\right) \cos ^{2} \theta \sin ^{2} \theta F_{v}\left(v_{1}, t\right) \\
& \cdot F_{v}\left(v_{2}, t\right) k\left(v_{1}, v_{2}, u\right) d v_{1} d v_{2} d u .
\end{aligned}
$$


Set

$$
K^{\prime}=4 K_{s} \int \cos \theta \sin \theta \beta(\theta) d u / C_{s} \int \cos ^{2} \theta \sin ^{2} \theta \beta(\theta) d u,
$$

and assume that $K^{\prime} \geqq 4$. The integrals in the right member of (4.2) over the set $\left\{\left(v_{1}, v_{2}, u\right) ;\left|v_{1}\right|>K^{\prime}\left|v_{2}\right|\right\}$ can be estimated from above by

$$
-(1 / 4) C_{s} \int\left(\left|v_{1}\right|^{s}+\left|v_{2}\right|^{s}\right) \cos ^{2} \theta \sin ^{2} \theta F_{v}\left(v_{1}, t\right) F_{v}\left(v_{2}, t\right) k\left(v_{1}, v_{2}, u\right) d v_{1} d v_{2} d u,
$$

where the integration is over the same set. The integrals in the right member of (4.2) over the set

$$
\left\{\left(v_{1}, v_{2}, u\right) ;\left|v_{2}\right|^{s-1}>K^{\prime}\left|v_{1}\right|^{s-1}\right\}
$$

can be estimated from above by

$$
-(1 / 4) C_{s} \int\left(\left|v_{1}\right|^{s}+\left|v_{2}\right|^{s}\right) \cos ^{2} \theta \sin ^{2} \theta F_{v}\left(v_{1}, t\right) F_{v}\left(v_{2}, t\right) k\left(v_{1}, v_{2}, u\right) d v_{1} d v_{2} d u,
$$

where the integration is over the same set. The integrals in the right member of (4.2) over the remaining part of $R^{6} \times B$ can be estimated by

$$
K_{2}\left(\int_{R^{3}}\left(1+v^{2}\right) F_{0}(v) d v\right)^{2}
$$

if $s \leqq 4-\lambda$, and otherwise by

$$
K_{2}\left(\int_{R^{3}}\left(1+|v|^{(s+\lambda) / 2}\right) F_{v}(v, t) d v\right)^{2} .
$$

And so by elementary computations, for $s \leqq 4-\lambda$,

$$
\begin{aligned}
& \partial_{t}\left(\int|v|^{s} F_{v}(v, t) d v\right) \leqq K_{3}\left(\int\left(1+v^{2}\right) F_{o}(v) d v\right)^{2} \\
& \quad-\frac{1}{4} C_{s} \int \cos ^{2} \theta \sin ^{2} \theta \beta(\theta) d u \int|v|^{s} F(v, t) d v \int F_{o}(v) d v .
\end{aligned}
$$

Here $K_{3}>0$ is independent of $v$ and $t$. For $s>4-\lambda\left(\int\left(1+v^{2}\right) F_{o}(v) d v\right)^{2}$ is substituted by $\left(\int\left(1+|v|^{(s+\lambda) / 2}\right) F_{v}(v, t) d v\right)^{2}$. From here we obtain the desired global bound

$$
\sup _{v \in N} \sup _{t} \int|v|^{s} F_{v}(v, t) d v<\infty
$$

by induction in the usual way (see e.g. [A1]).

By transfer the above also holds in the non-standard context. There, in particular, $F_{v}$ of Proposition 4.1 satisfies Lemma 4.3 for $v \in^{*} N-N$, and also

$$
\int_{R^{3}}^{o} F_{v}(v, t)^{*} g(v)^{*} d v=\int F(v, t) g(v) d v \quad\left(g \in L^{\infty}\left(R^{3}\right)\right)
$$

Notice that the case $\varepsilon=1$ transforms into the case of general $\varepsilon>0$ after the substitution

$$
t \rightarrow \varepsilon t, F_{\nu \varepsilon}(v, t)=F_{v}(v, t / \varepsilon) .
$$

Here the arguments of Sect. 3 imply, that for $\varepsilon \approx 0$ there are for Loeb a.e. $t \in{ }^{*} R_{+} a(t)$, $b(t) \in R_{+}$, and $c(t) \in R^{3}$, such that the solution $F_{v \varepsilon}$ satisfies

$$
F_{v \varepsilon}(v, t) \approx a(t) \exp \left(-b(t) v^{2}+c(t) \cdot v\right)
$$


for Loeb a.e. $v \in n s^{*} R^{3}$. But since $F_{v \varepsilon}$ has globally bounded moments of the order $s(>2)$ also for $\varepsilon \approx 0$, it follows that $v^{2} F_{v \varepsilon}(v, t)$ is $S$-integrable for $t \in^{*} R_{+}$as a function of $v$, and that the functions $a(t), b(t)$ and $c(t)$ are independent of $t$ and $\varepsilon, a(t)=a$, $b(t)=b, c(t)=c$. The constants $a, b \in R$, and $c \in R^{3}$ are uniquely determined by

$$
\begin{aligned}
\int F_{o}(v) d v & =\int a \exp \left(-b v^{2}+c \cdot v\right) d v, \\
\int v F_{o}(v) d v & =\int v a \exp \left(-b v^{2}+c \cdot v\right) d v, \\
\int v^{2} F_{o}(v) d v & =\int v^{2} a \exp \left(-b v^{2}+c \cdot v\right) d v .
\end{aligned}
$$

Below we write the corresponding Maxwellian as $M(v)=a \exp \left(-b v^{2}+c \cdot v\right)$. This proves

Theorem 4.4. For $\varepsilon \approx 0, v \in \in^{*} N-N$, and Loeb a.e. $t \in^{*} R_{+}$,

$$
F_{v \varepsilon}(v, t) \approx M(v) \text { for Loeb a.e. } v \in n s^{*} R^{3} .
$$

Theorem 4.4 can be used to study the asymptotic behaviour of the solution $F$ of the space-homogeneous Boltzmann equation (4.1), given in Proposition 4.1.

Theorem 4.5. Assume that

$$
F_{o} \ln F_{o} \in L^{1}\left(R^{3}\right),\left(1+|v|^{s}\right) F_{o} \in L_{+}^{1}\left(R^{3}\right) \text { for some } s>2 .
$$

Then the solution $F$ of (4.1) given by Proposition 4.1 satisfies

$$
\lim _{t \rightarrow \infty} \int_{R^{3}}|F(v, t)-M(v)| d v=0 .
$$

Proof. If the theorem does not hold, then for some $\eta \in R_{+}$, there is a sequence $\left(t_{j}\right)_{j \in N}$ of reals increasing to infinity, such that

With

$$
\int_{R^{3}}\left|F\left(v, t_{j}\right)-M(v)\right| d v>\eta .
$$

$$
\chi_{j}=\operatorname{sign}\left(F\left(v, t_{j}\right)-M(v)\right) \in L^{\infty}\left(R^{3}\right),
$$

this can be written

$$
\int_{R^{3}}\left(F\left(v, t_{j}\right)-M(v)\right) \chi_{j}(v) d v>\eta .
$$

It follows that for $v \in^{*} N-N$

$$
\int_{R^{3}}\left|F_{v}\left(v, t_{j}\right)-{ }^{*} M(v)\right|^{*} d v \geqq \int\left(F_{v}\left(v, t_{j}\right)-{ }^{*} M(v)\right)^{*} \chi_{j}(v)^{*} d v>\eta
$$

By the uniform $S$-continuity of $F_{v}:{ }^{*} R_{+} \rightarrow{ }^{*} L_{+}^{1}\left(R^{3}\right)$, there is $\delta \in R_{+}$, such that

$$
\int_{R^{3}}\left|F_{v}(v, t)-{ }^{*} M(v)\right|^{*} d v>\eta / 2
$$

for $t \in *\left[t_{j}-\delta, t_{j}+\delta\right], j \in N$. Moreover the sequence $\left(t_{j}\right)_{j \in N}$ can be extended to an increasing internal sequence $\left(t_{j}\right)_{j=1}^{j^{\prime}}, j^{\prime} \in * N-N$ for which (4.3) holds. But $2 j^{\prime} \delta=\tau$ is 
not finite. It follows that for $\varepsilon=1 / \tau \approx 0$, and $T=t_{j^{\prime}}+1$,

$$
\left\{t \in[0, T \varepsilon] ; \int_{R^{3}}\left|F_{v \varepsilon}(v, t)-{ }^{*} M(v)\right|^{*} d x d v>\eta / 2\right\}
$$

has $*$ Lebesgue measure not smaller than one.

This gives a contradiction, since by Theorem 4.4, for Loeb a.e. $t \in R_{+}$,

$$
\int_{R^{3}}^{o}\left|F_{v \varepsilon}(v, t)-{ }^{*} M(v)\right| * d x d v=\int_{n s^{*} R^{3}}\left|{ }^{o} F_{v \varepsilon}(v, t)-{ }^{o *} M(v)\right| L d x d v=0 .
$$

Hence the theorem holds.

The $|v|^{s}$-moment of $F$ is globally bounded in time, which gives the following corollary to Theorem 4.5 .

Corollary 4.6. $\lim _{t \rightarrow \infty} \int_{R^{3}}|v|^{s^{\prime}}|F(v, t)-M(v)| d v=0$ for $0<s^{\prime}<s$.

\section{References}

[AFHL] Albeverio, S., Fenstad, J. E., H $\phi$ egh-Krohn, R., Lindstr $\phi$ m, T.: Nonstandard methods in stochastic analysis and mathematical physics. New York: Academic Press 1986

[A1] Arkeryd, L.: On the Boltzmann equation. Arch. Ration. Mech. Anal. 45, 1-34 (1972)

[A2] Arkeryd, L.: An existence theorem for a modified space-inhomogeneous, non-linear Boltzmann equation. Bull. Am. Math. Soc. 78, 610-614 (1972)

[A3] Arkeryd, L.: Loeb solutions of the Boltzmann equation. Arch. Ration. Mech. Anal. 86, 85-97 (1984)

[C] Cercignani, C.: Theory and application of the Boltzmann equation. New York: Academic Press 1975

[E] Elmroth, T.: The Boltzmann equation; on existence and qualitative properties. Dissertation, Chalmers University of Technology 1984

[L] Loeb, P. A.: Conversion from non-standard measure spaces and applications in probability theory. Trans. Am. Math. Soc. 211, 113-122 (1975)

[TM] Truesdell, C., Muncaster, R. G.: Fundamentals of Maxwell's kinetic theory of a simple monatomic gas. New York: Academic Press 1980

Communicated by J. L. Lebowitz

Received August 2, 1985 
